\title{
ВЗАИМОДЕЙСТВУЕТ ЛИ РЕАЛЬНАЯ ФОРМА С ИДЕАЛЬНОЙ? ИССЛЕДОВАНИЕ ОВЛАДЕНИЯ СЧЕТОМ НА ЧИСЛОВОЙ ПРЯМОЙ С ПОМОЩЬЮ ЗАПИСИ ДВИЖЕНИЙ ГЛАЗ
}

\author{
A.Ю. ШВАРЦа, \\ ${ }^{a}$ Утрехтский Университет, 3584 СС, Нидерланды, Утрехт, ул. Принстонпляйн, д. 5 \\ ${ }^{b}$ Московский государственный университет имени М.В. Ломоносова, 119991, Россия, Москва, \\ Ленинские горы, Ә. 1
}

\begin{abstract}
Резюме
В статье рассматривается проблема овладения математическим знанием на примере обучения дошкольников счету на числовой прямой. С помощью записи движений глаз раскрывается многообразие стратегий определения числа на числовой прямой. Показано существенное различие между стратегиями, используемыми взрослыми, стратегиями, используемыми в процессе обучения, и стратегиями детей после обучения $\left(\chi^{2}=44.936 ; p<\right.$ 0.001). Выявленные различия в стратегиях по ряду параметров (направление пересчета вверх или вниз по числовой прямой, положение целевой точки в начале или в конце счета) свидетельствуют о том, что самостоятельные решения детей после обучения в большей степени похожи на решения задач взрослыми, чем стратегии, предложенные при обучении. Анализ данных о движениях глаз и видео совместной деятельности родителей и детей в ходе формирующего эксперимента показывают, что взрослые формируют способ восприятия числовой прямой детьми с помощью жестикуляции и синхронизированных с ней вербальных указаний. Однако родители не раскрывают в ходе обучения идеальную форму, т.е. многообразие стратегий, характерное для взрослого восприятия, а выстраивают базовую, учебную форму действия, надежно доступную ребенку. Несмотря на это, дети самостоятельно дополняют предложенную взрослыми базовую стратегию пересчета другими стратегиями, основываясь на интегральном представлении о числе. Недоступной оказывается лишь стратегия, требующая принципиально иного способа действия: рассмотрения числовой прямой как системы равных интервалов, а не последовательного ряда чисел. Согласно результатам нашего исследования, идеальная, культурная форма восприятия существует в скрытом виде, ребенку необходимо самостоятельно переоткрывать ее, как используя предложенную взрослым базовую стратегию пересчета, так и встраивая конкретную задачу в целостную систему знаний.
\end{abstract}

Ключевые слова: культурно-исторический подход, Выготский, математическое образование, обучение, идеальная форма, совместная деятельность, числовая прямая, математическое понятие, движения глаз, окулография.

Работа выполнена при финансовой поддержке РФФИ, проект № 15-06-06319 «Развитие восприятия визуального математического материала в интерсубъективном взаимодействии». 
Исследование математических способностей и обучения математике находится на стыке психологии развития, когнитивной психологии и наук об образовании. Интрига совмещения проблематики и инструментария этих областей в том, что результаты такого совмещения одновременно проясняют фундаментальные закономерности человеческого развития и позволяют делать конкретно-практические выводы, способствующие совершенствованию образовательного процесса. Согласно современным представлениям (Duval, 2006; Hitt, 1998; Presmeg et al., 2016; Radford, 2010; и мн. др.), математическое знание является мультимодальным и формируется как единство множества моделей или репрезентаций, представленных в формальной, вербальной, визуальной, тактильной форме. Наше исследование посвящено изучению процессов овладения одной из таких репрезентаций понятия числа числовой прямой. Формирующий эксперимент в духе Л.С. Выготского позволяет описать процесс развития и приблизиться к пониманию значения взаимодействия различных компонентов понятия числа в обучении.

\section{Пространственные представления о числовой прямой в составе понятия числа}

Согласно данным экспериментальных и клинических исследований, пространственные представления являются существенной составляющей понятия числа, а также того, что принято называть чувством числа (number sence). Результаты исследований А.Р. Лурии (1962) показывают, что арифметические операции разрушаются при поражении теменно-затылочных долей мозга, ответственных за пространственные представления. Пациенты с такими поражениями путают зрительно схожие числа (такие как 69 и 96), а также затрудняются в понимании разрядного строения чисел. Кроме того, возникают ошибки, вызванные разрушением представления о направлении счета, т.е. о последовательном и направленном расположении чисел в числовом ряду и на числовой прямой. Так, при вычитании с переходом через десяток они могут вычесть до десятка, а потом прибавить, двинувшись в другую сторону (например, вычисляя 31-7, они считают 30-7 и потом вычитают еще 1 вместо того, чтобы прибавить, и получают 22 вместо 24$)$.

Современные исследования с использованием методов нейровизуализации подтверждают эти данные и показывают, что активация внутритеменной борозды связана с различными арифметическими операциями. В частности, латеральная часть этой области мозга, вероятно, выполняет функцию прослеживания вдоль числовой прямой, сходную с прослеживанием на визуальной сцене при оперировании другими мысленными образами (Hubbard et al., 2005).

С этим выводом согласуются данные поведенческих экспериментов. Феномен, известный как SNARC-эффект (Dehaene et al., 1993), заключается в том, что испытуемые отчитываются о маленьких числах быстрее, если числа (или вербальная запись числа - Fias, 1996) предъявлены в левое полуполе, и быстрее о больших числах, если они предъявлены в правое полуполе. Предполагается, что, даже решая задачу, не требующую активации представления 
о величине числа, испытуемые связывают число с его положением на числовой прямой или в числовом ряду. Аналогичные данные были получены при анализе поворота глаз испытуемыми в темной комнате, т.е. при отсутствии внешней зрительной стимуляции. Решая задачу на нахождение числа посередине между двумя числами, они совершали горизонтальные саккады, причем если первое названное число было больше, то саккады совершались преимущественно справа налево, как будто двигаясь к среднему по мысленной числовой прямой (Loetscher et al., 2008).

\section{Возникновение представлений о числовой прямой в онтогенезе}

Каким образом возникают пространственные представления о числе? Одним из механизмов является освоение числовой прямой - одной из внешних визуальных репрезентаций, или моделей, понятия числа. Серия исследований об оценке положения числа на числовой прямой вскрывает основные стратегии и очередность их появления в ходе обучения.

В целом выделяются такие стратегии, как счет от начальной точки, счет от конечной точки и счет от середины отрезка.

Первыми в онтогенезе появляются стратегии отсчета от начальной или конечной точки. Способность уже первоклассников использовать конечные точки показана в ряде исследований (Link et al., 2014; Petitto, 1990; Schneider et al., 2008; и др.). Согласно выводам некоторых авторов (Schneider et al., 2008), дети, уже начиная с первого класса, также могут использовать и серединную точку для нахождения числа на числовой оси, согласно другим данным (Petitto, 1990; Siegler, Opfer, 2003), первоклассники не используют серединную точку.

Более детальный анализ когнитивных операций, лежащих за представлениями о числовой прямой, в отличие от числового ряда, показывает, что использование срединной точки содержит представление не только о последовательном расположении чисел слева направо, но также об относительных расстояниях между числами. Такой сдвиг от представления о последовательности к представлению о пропорциональном соотношении (sequence-to-proportion shift) наблюдается только к третьему классу ${ }^{1}$, когда дети для оценки положения числа на отрезке числовой прямой начинают использовать срединную точку, опираясь на равенство интервалов от нее до концов отрезка (Petitto, 1990).

Другой объяснительной конструкцией по сути того же феномена является предположение, что мысленное представление о логарифмическом расположении чисел на числовой прямой постепенно сменяется адекватным линейным представлением. Так, если в втором и четвертом классе превалирует скопление чисел в начале числовой оси (как если бы использовалась логарифмическая линейка для оценки длины), то к шестому классу это проходит и числа оказываются распределены равномерно, то же наблюдается и у взрослых испытуемых (Siegler, Opfer, 2003).

\footnotetext{
${ }^{1}$ Третий класс в США посещают дети 8-9 лет.
} 
В ряде работ используется метод регистрации движений глаз при выполнении заданий на оценку положения числа на числовой прямой. Согласно этим данным, дети уже начиная с первого класса чаще фокусируются на концах отрезка числовой прямой, а также на срединной точке, используя все три положения как точки ориентации. При этом превалирующей стратегией является счет от 0 или от середины до целевой точки (Schneider et al., 2008). Данные о движениях глаз, полученные той же группой исследователей (Heine et al., 2010), свидетельствуют о линейных представлениях о расположении чисел на числовой прямой уже в первом классе, несмотря на соответствие поведенческих данных логарифмической модели. Можно говорить, что данные о движениях глаз вскрывают раннее созревание определенных операций, которое может еще не проявляться в итоговом ответе и обнаруживаться в поведенческих данных только более старших возрастных групп. Это соответствует данным С. Голдин-Медоу (см., например: Goldin-Meadow, 1999): телесная (embodiment) подготовка математических знаний и навыков предвосхищает их эксплицитное проявление при непосредственном тестировании.

Как можно видеть, большинство исследований посвящено изучению числовой прямой как уже сформированному ментальному образу, позволяющему выносить некоторые суждения о числах. Нас интересуют механизмы возникновения этого образа и стратегий работы с ним.

Как указывает Л.С. Выготский, «величайшая особенность детского развития заключается в том, что это развитие совершается в таких условиях взаимодействия со средой, когда идеальная форма, конечная форма... реально взаимодействует, реально оказывает влияние на первичную форму, на первые шаги детского развития» (Выготский, 2001, с. 83-84). Идеальная форма - это не записанные правила или определения, хранящие культурное достояние, но сами формы поведения, деятельности, существующие в культуре, окружающей ребенка, такие как речевая деятельность, оперирование бытовыми предметами и знаковыми системами со стороны взрослых. В приложении к освоению математического знания к идеальной форме следует отнести математические действия, в частности, действие пересчета и другие способы количественной оценки, присущие культурному поведению взрослых.

Каким образом происходит усвоение этой идеальной формы? Согласно Выготскому, идеальная форма усваивается в ходе совместной деятельности со взрослым, который раскрывает ее в интерпсихическом взаимодействии с ребенком. В психологии математического образования значительное внимание уделяется микроанализу процессов этой совместной деятельности, в которой ребенок научается воспринимать математические изображения и знаки так же, как взрослый. Л. Радфорд (Radford, 2010) вслед за К. Марксом рассматривает этот процесс как «окультуривание» (domestication) органов чувств в ходе социальной практики. Исследуя видео- и аудиозаписи совместной деятельности учителя и ученика, Радфорд показывает, как все семиотические регистры (визуальная репрезентация, жесты, интонации, их ритм и др.) соединяются вместе, позволяя ребенку наполнить визуальную репрезентацию смыслом, выделить ее существенные аспекты и объективировать (objectification) 
математическое содержание изображения. B.-M. Рош (Roth, 2008) детально анализирует, как просодические характеристики речи направляют внимание слушающего и как при этом внимание к одним и тем же частям диаграммы может сопровождаться различными жестами, делающими наиболее выпуклыми необходимые аспекты математического смысла. Р. Бюланд (Bjuland, 2012) подчеркивает значение жестов учителя, включенных в мультимодальную коммуникацию, для формирования ранних алгебраических представлений у учеников, а также показывает, как жесты одного ученика становятся средством переосмысления задачи для другого ученика.

Недостатком приведенных исследований является отсутствие инструмента для непосредственного анализа перцептивных процессов ребенка. О том, что культурный способ восприятия был усвоен, исследователи судят по решению задач и успешной коммуникации с учителем или сверстником. В нашем исследовании с помощью записи движений глаз мы непосредственно проанализировали используемые ребенком стратегии счета на числовой прямой, соотнесли их со стратегиями взрослых и стратегиями, разворачивающимися в интерпсихическом пространстве совместной деятельности.

\section{Эмпирическое исследование формирования культурного способа восприятия числовой прямой}

Общей целью эмпирического исследования было изучение механизмов передачи идеальной формы от взрослого к ребенку на примере обучения родителями детей культурному способу действия, а именно счету на числовой прямой.

Конкретными задачами исследования являлись:

1) раскрытие стратегий счета на числовой прямой у взрослых;

2) анализ стратегий ребенка в ходе совместной деятельности ребенка со взрослым в ходе обучения;

3) выявление стратегий восприятия числовой прямой детьми при решении задач самостоятельно после обучения;

4) анализ жестов, интонаций и вербальных указаний взрослых, направленных на трансформацию операциональной стороны восприятия ребенка, выявление стратегий, использованных в обучении;

5) сопоставление стратегий, присущих взрослым и детям, а также стратегий, актуализированных в ходе обучения.

Исследование выполнено с помощью качественно-количественного анализа глазодвигательной активности детей, синхронизированной с видео- и аудиозаписью жестов и речи детей и родителей. На первом этапе, в ходе качественного анализа, были выделены стратегии восприятия числа на числовой прямой, а на втором этапе статистические процедуры позволили сопоставить применение выделенных стратегий разными группами испытуемых. 


\section{Методика}

Испьтуемые. В исследовании приняли участие шесть пар родителей и детей-дошкольников в возрасте от 5.3 до 6.8 лет. Участвовали двое пап и четыре мамы, два мальчика и четыре девочки. (Для удобства, в ходе качественного анализа мы будем называть каждую пару буквами А, Б, В, Г, Д, Е - по порядку участия в исследовании.) Возраст детей выбирался исходя из цели изучения процесса развития, т.е. такой, в котором освоение числовой прямой находилось в зоне ближайшего развития детей. Никто из них не видел числовую прямую до исследования и не знал, как самостоятельно решить экспериментальную задачу. За участие в исследовании детям давалось печенье Барни.

Небольшое количество испытуемых определялось трудоемкостью качественного анализа, потребовавшего для оценки стратегий восприятия ручного анализа всех фиксаций испытуемых.

Процедура и материалы исследования. Всего испытуемым предлагалось решить три вида задач на счет в пределах 10, задача каждого типа предъявлялась восемь раз с разными ответами (от 2 до 9). Основной задачей был счет на числовой оси.

На оси, изображенной в интервале от 0 до 10 (см. рисунки 1, 2, стимульный материал предоставлен образовательной платформой Uchi.ru), каждое целое число было отмечено риской. Были подписаны числа 0,5 и 10 , они отмечались более жирными рисками. Такая числовая ось существенно отличается от принятой в исследованиях стратегии оценки положения числа на числовой оси. Во всех известных нам работах (Heine et al., 2010; Link et al., 2014; Petitto, 1990; Schneider et al., 2008; Siegler, Opfer, 2003; Sullivan et al., 2011; и др.) в качестве числовой прямой используется горизонтальный сплошной отрезок с рисками и подписями чисел только на концах. Выбор нами более математически нагруженной визуальной модели обусловлен следующим. В большинстве из этих работ исследуется внутреннее, ментальное представление о числе и возможность трансформировать его в пространственные соотношения. Целью же нашей работы было изучить процессы становления культурного восприятия внешней визуальной модели числа - числовой оси, поэтому наш стимульный материал более полно отражал систему отношений, стоящую за пониманием числового ряда в пределах десяти.

В большинстве работ исследуется восприятие и процессы оценки взрослых (Link et al., 2014; Sullivan et al., 2011; и др.) и школьников с первого по шестой класс (Link et al., 2014; Schneider et al., 2008; и др.), чаще используется ось от 0 до 100 (Heine et al., 2010; Schneider et al., 2008), но в некоторых работах - ось до 10 (Link et al., 2014), до 1000 (Sullivan et al., 2011) или даже до 10000 (Link et al., 2014). Нас же интересовала ситуация, когда дети еще заведомо не сталкивались с числовой прямой в ходе формального обучения, поэтому нашими испытуемыми стали дошкольники; однако для того, чтобы задача находилась в их зоне ближайшего развития, мы выбрали числовую прямую от 0 до 10. 
Испытуемым предъявлялись числовая ось, над одной из рисок которой располагался кузнечик. Наверху того же слайда предъявлялось задание: «На какой точке сидит кузнечик?» Ответ давался нажатием цифры на клавиатуре. В том случае, когда число 5 было целевым, соответствующая риска не была подписана.

Использовалось две интерферирующих задачи: счет бусин, расположенных на экране в хаотичном порядке, и выбор банки с указанным количеством фруктов из трех банок, предъявленных на экране. Таким образом интерферирующие задачи также задействовали операцию счета, но не использовали визуальную модель «Числовая ось» и не актуализировали операции счета на ней.

Процедура проведения исследования для каждой диады была следующей.

На первом этапе производилась запись движений глаз родителя, который сначала решал основную задачу, а потом две интерферирующие. Таким образом мы получали информацию о стратегиях счета у взрослых. Во время этого этапа ребенок знакомился с процедурой калибровки и записи движений глаз, а в процессе прохождения задач родителем занимался складыванием пазла и не имел возможности предварительно ознакомиться с задачами.

На втором этапе к исследованию приглашались оба участника: ребенок находился перед монитором для записи глазодвигательной активности, а взрослый садился рядом. Взрослому давалась инструкция: «Помогите ребенку решить задачи. Чувствуйте себя свободно. Вы можете прерывать ребенка в любой момент и давать дополнительные разъяснения». Таким образом у взрослого создавалась установка на активное взаимодействие с ребенком. Необходимость такого достаточно настойчивого побуждения взрослого к активному вмешательству в процесс решения задач была обусловлена результатами пилотажа, который показал, что иначе родители склонны предоставлять детей самим себе и минимально вмешиваться в процесс обучения.

На третьем этапе производилась регистрация движений глаз при самостоятельном решении задач ребенком. Сначала ребенок решал интерферирующие задачи, а затем переходил к основной, решая ее повторно после обучения родителем.

\section{Annapamypa}

Запись движений глаз проводилась с помощью установки SMI RED c частотой регистрации 120 Гц. Положение головы участников фиксировалось с помощью подбородника, чтобы облегчить детям процесс калибровки и необходимость удерживать голову в достаточно узком коридоре, позволяющем осуществлять запись движений глаз. Использовалась пятиточечная калибровка. Экран (21 дюйм) располагался на расстоянии 45 см.

Взаимодействие ребенка и родителя записывалось на внешнюю видеокамеру с частотой 30 Гц. Стимуляция подавалась с помощью программы Experiment Center 3.1 с подключаемым пакетом Observational package для записи взаимодействия на внешнюю камеру. Для записи глазодвигательной 
активности использовался iViewX 3.1, качественный анализ результатов проводился в программе Begaze 3.1, также использовалось собственное программное обеспечение, написанное в Mathlab 2014b и позволяющее более точно синхронизировать данные внешней видеокамеры и данные о движениях глаз. Для статистического анализа использовался пакет SPSS 20.0.0.

\section{Результаты и их обсуждение}

\section{Стратегии счета взрослых и детей: идеальная форма и ее усвоение}

Как показали решения интерферирующих задач, все наши испытуемые уже владели числовым рядом и были способны к последовательному пересчету отдельных объектов, делая ошибки лишь в редких случаях. Следовательно, в ходе прохождения основной серии задачей являлась именно координация числового ряда и незнакомой визуальной модели, а не усвоение порядка счета.

Качественный анализ записей движений глаз небольшого количества испытуемых позволил нам оценить используемый способ действия в каждой пробе, что рассматривается как перспективный, но трудоемкий шаг (Schneider et al., 2008). Запись движений глаз одного из родителей не была произведена вследствие его косоглазия. В некоторых случаях (в 4 пробах из 136) выявление способа действия было затруднительно из-за потери взора, такие пробы не учитывались. Таким образом, мы получили данные о способах перцептивных действий взрослых, детей при решении задачи совместно со взрослыми и детей при самостоятельном решении. В ряде случаев испытуемые использовали сразу две стратегии, вероятно, проверяя свой результат, тогда мы засчитывали обе.

Опишем стратегии, которые использовались взрослыми и детьми для определения числа на числовой прямой (таблица 1 содержит примеры сырых данных о движении глаз, соответствующих определенным стратегиям). В целом они повторяют стратегии, указанные в работе Петитто (Petitto, 1990), но имеется существенное расширение.

Большинство стратегий связаны с пересчетом рисок на числовой оси. Однако риски можно считать разными способами: можно считать от 0 до целевой точки, это всегда будет счет по числовой оси «вверх», в порядке, соответствующем числовому ряду (стратегия 1). Другой стратегией будет счет от целевой точки до числа 0, это будет счет «вниз» (стратегия 2). Результат получится один и тот же, но ребенку придется применить нетривиальное знание, что результат счета не зависит от порядка пересчета (Gelman, Gallistel, 1978).

Кроме того, можно пользоваться числом 10, обозначенным на конце отрезка числовой прямой, и посчитать, сколько делений от целевой точки до числа 10 (стратегия 3, это будет счет «вверх»), или посчитать, сколько делений будет от числа 10 до целевой точки (стратегия 4, счет «вниз»). Кроме того, можно использовать серединную точку. В данных были представлены три из 


\section{Стратегии определения числа на числовой прямой}

\begin{tabular}{|c|c|c|c|c|c|}
\hline № & Стратегия & Пример сырых данных о движениях глаз & $\mathbf{P}$ & до & Д \\
\hline 1 & От 0 вверх к & $x_{10+\infty}+5$ & 1 & 23 & 17 \\
\hline & & & & & \\
\hline 2 & $\begin{array}{c}\text { От целевой точки } \\
\text { вниз к } 0\end{array}$ & 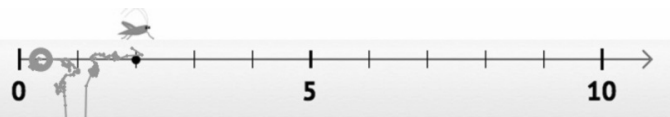 & 8 & 4 & 9 \\
\hline 3 & $\begin{array}{l}\text { От целевой точки } \\
\text { вверх к } 10\end{array}$ & 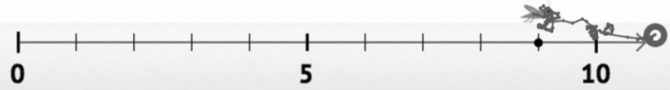 & 2 & 6 & 5 \\
\hline 4 & $\begin{array}{l}\text { От } 10 \text { вниз до } \\
\text { целевой точки }\end{array}$ & $1,1,1, \bigcap_{5},+\frac{1}{10}$ & 4 & 8 & 4 \\
\hline 5 & $\begin{array}{l}\text { От } 5 \text { вверх к } \\
\text { целевой точке }\end{array}$ & $\left.\right|_{0}$ & 7 & 12 & 7 \\
\hline 6 & $\begin{array}{c}\text { От } 5 \text { вниз к } \\
\text { целевой точке }\end{array}$ & ${ }_{0}, 1, \frac{1}{5}, 1,1, \frac{1}{10}$ & 6 & 9 & 7 \\
\hline 7 & $\begin{array}{c}\text { От целевой точки } \\
\text { вниз к } 5\end{array}$ & 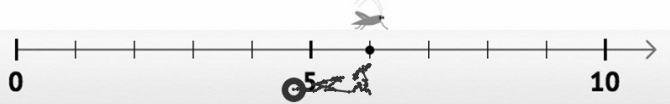 & 3 & 0 & 5 \\
\hline 8 & $\begin{array}{l}\text { Сопоставление с } \\
\text { предыдущим } \\
\text { ответом }\end{array}$ & ${ }_{0}, 1,1,1, \frac{1}{5}$ ats & 3 & 0 & 1 \\
\hline 9 & $\begin{array}{c}\text { Оценка } \\
\text { расстояния до } \\
\text { ближайших точек }\end{array}$ & $\underset{0}{1+1+1,+1, ~}$ & 5 & 1 & 0 \\
\hline 10 & $\begin{array}{l}\text { Ответ за одну } \\
\text { фиксацию }\end{array}$ & & 6 & 2 & 2 \\
\hline
\end{tabular}

Примечание. $\mathrm{P}$ - родители, ДО - дети при обучении, Д - дети. 
возможных четырех стратегий, опирающихся на число 5: счет от числа 5 к целевой точке «вверх» и «вниз» в зависимости от того, было ли целевое число больше или меньше пяти (стратегии 5 и 6), а также счет от целевой точки к числу 5 «вниз» для чисел больше пяти (стратегия 7). Для целевых чисел меньше 5 счет вверх от целевой точки до числа 5 является, по всей видимости, нецелесообразным.

Стратегии сходны с указанными Петитто (Petitto, 1990) по ориентационным точкам. Однако такие стратегии характеризуются непосредственным пересчетом на числовой оси, используемым довольно редко при оценке положения числа на числовой прямой без делений (Link et al., 2014). Нами исследуются несколько другие процессы: не приблизительной оценки положения числа на числовой прямой, а точного вычисления. Стратегии сходны в общих чертах, но отличаются по конкретной операциональной реализации. Кроме того, нам удалось детальнее описать существующие стратегии за счет качественного анализа записей движений глаз отдельных субъектов, тогда как в существующих работах анализируются лишь частота фиксации в разных частях числовой оси и точность полученной оценки, а на основе этого делаются предположения о стратегии.

Таким образом, по своему операциональному составу действия вычисления варьировались по следующим характеристикам:

1) точка ориентира (orientational point) (0 - стратегии 1 и 2; 10 - стратегии 3 и 4; 5 - стратегии 5, 6 и 7);

2) направление пересчета (вверх по числовой оси - стратегии 1, 3, 5; вниз по числовой оси - стратегии $2,4,6,7)$;

3 ) позиция целевой точки в пересчете (счет до целевой точки - стратегии $1,4,5,6$; счет от целевой точки к точке ориентира - стратегии $2,3,7$ ).

В некоторых случаях испытуемые использовали в качестве точки ориентира целевую точку предыдущего задания (стратегия 8).

Помимо стратегий пересчета, нам удалось выявить принципиально другую стратегию, она применялась для целевых точек 5 и 8 (стратегия 9). Испытуемый смотрел на целевую точку, а затем быстро, зачастую даже без фиксаций, переводил взгляд к соседним ориентационным точкам. По всей видимости, эта стратегия связана с оценкой относительных расстояний от точек-ориентиров до целевой.

Еще один вариант глазодвигательной активности - это ответ после единственной фиксации на целевом числе (стратегия 10). В данном случае данные о движении глаз не дают оснований для выявления стратегии, но можно предположить, что она не требовала пересчета. Эта стратегия встречалась для целевых чисел 2, 6 и 9, т.е. близких к точкам ориентира, а также для вопроса о числе 5, где испытуемые могли ориентироваться на выделенную риску.

Мы сравнивали частоту встречаемости разных стратегий у родителей, детей под руководством родителей и детей, решавших задачи самостоятельно. Анализ с помощью таблиц сопряженности показал, что имеются существенные различия в предпочитаемых стратегиях $\left(\chi^{2}=44.936 ; p<0.001\right)$. 
Как видно из таблицы 1, отражающей, сколько раз была использована каждая стратегия, дети преимущественно пользовались стратегиями с пересчетом. Стратегия 9 была доступна практически только взрослым. Это согласуется с данными исследований о том, что дети только к третьему классу начинают понимать числовую прямую как отражающую расстояния между числами, лишь тогда они начинают использовать стратегию пропорциональной оценки расстояний (Link et al., 2014), которая у взрослых становится доминирующей (Sullivan et al., 2011).

Далее мы сравнили различия между группами для разных стратегий пересчета, разделив их по указанным выше факторам: 1) точка ориентира, 2) направление пересчета, 3) позиция целевой точки.

Не выявлено статистически значимых различий в использовании чисел 0,5 или 10 в качестве точек ориентира взрослыми и детьми дошкольного возраста. Согласно литературным данным, уже первоклассники способны использовать конечные точки (Link et al., 2014; Petitto, 1990; Schneider et al., 2008; и др.). Мы дополнили эти данные информацией о дошкольниках. Мнения о доступности использования серединной точки в литературе расходятся (Link et al., 2014; Petitto, 1990; Schneider et al., 2008; Siegler, Opfer, 2003). Однако в нашем случае серединная точка была специально выделена и даже подписана, вероятно, поэтому ее использование оказалось доступно дошкольникам.

По двум другим факторам различия наблюдаются. Так, стратегии, используемые в разных сериях, различаются по направлению пересчета $\left(\chi^{2}=9.576 ; p=0.008\right)$. Как видно из таблицы 2, взрослые чаще считают «вниз» по числовой оси (если совершается пересчет, то он делается вверх только в 32.3\% случаев), тогда как дети при обучении чаще считают «вверх» (66.1\%). Когда же дети считают самостоятельно, то они занимают промежуточное положение - считают «вверх» в 53\% случаев, что отличается от стратегий взрослых на уровне тенденции $\left(\chi^{2}=9.576 ; p=0.056\right)$.

Это выявляет интересный факт: поведение детей при самостоятельном решении в большей степени соответствует идеальной форме, наблюдаемой у взрослых, чем то поведение, которое дети показывают в процессе обучения. В предположении, что в процессе обучения взрослые передают ту самою идеальную форму, те способы счета, которыми обладают сами, этот результат выглядит парадоксально. Природа этого факта раскрывается ниже при анализе стратегий, выбираемых взрослыми для обучения.

Также обнаруживаются значимые различия в позищи целевой точки $\left(\chi^{2}=8.574\right.$; $p=0.014)$. Как видно из таблицы 3, взрослые пользуются как стратегией счета к целевому числу (58.1\%), так и стратегией счета от целевого числа к точке ориентира (41.9\%), однако в процессе обучения дети используют стратегию счета до целевого числа, т.е., например, «0-1-2-3-4!» или «5-6-7!» в 83.9 \% случаев, а стратегию счета от целевой точки до точки ориентира только в $16.1 \%$ случаев. Как и в случае с направлением пересчета, самостоятельные решения детей в большей степени похожи на стратегии родителей (64.8\% при счете к целевой точке и $35.2 \%$ при счете от целевой точке) и статистически от них не отличаются. 
Направление счета в разных группах

\begin{tabular}{|l|c|c|}
\hline & $\begin{array}{c}\text { Счет «вниз» } \\
\text { (справа налево) }\end{array}$ & $\begin{array}{c}\text { Счет «вверх» } \\
\text { (слева направо) }\end{array}$ \\
\hline Взрослые & $67.7 \%$ & $32.3 \%$ \\
\hline Дети на стадии обучения & $33.9 \%$ & $66.1 \%$ \\
\hline Дети & $46.3 \%$ & $53.7 \%$ \\
\hline
\end{tabular}

Таблица 3

Позиция целевой точки при счете в разных группах

\begin{tabular}{|l|c|c|}
\hline & $\begin{array}{c}\text { Счет от целевой точки к } \\
\text { ориентационной }\end{array}$ & $\begin{array}{c}\text { Счет от ориентационной } \\
\text { точки к целевой }\end{array}$ \\
\hline Взрослые & $41.9 \%$ & $58.1 \%$ \\
\hline Дети на стадии обучения & $16.1 \%$ & $83.9 \%$ \\
\hline Дети & $35.2 \%$ & $64.8 \%$ \\
\hline
\end{tabular}

Таким образом, дети, как и взрослые, свободно пользуются всеми тремя точками-ориентирами: 0,5 и 10. При этом взрослые предпочитают считать от точки-ориентира $к$ целевой точке, у детей в ходе обучения это предпочтение выражено в крайне сильной форме, а при самостоятельном решении задачи дети предпочитают считать к целевой точке в меньшей степени, чем при обучении, и их стратегии больше похожи на стратегии родителей. Та же закономерность выявляется при анализе направления счета: взрослые предпочитают считать вниз по числовой прямой, дети при обучении, напротив, предпочитают считать вверх, а при самостоятельном счете их способы действия опять же оказываются ближе к стратегиям взрослых, чем к продемонстрированным при обучении. Таким образом, способы действия, заложенные в обучении, не только не совпадают со способами действий взрослых, идеальной формой, но и отличаются от нее больше, чем способ действия, к которому приходят дети в итоге, встречаясь с задачей один на один.

\section{Процесс обучения: совместная деятельность и усвоение идеальной формы}

Какие же стратегии использовали взрослые при обучении детей? Следует отметить, что дети довольно легко справлялись с заданием, поэтому взрослые в большинстве случаев давали пояснения только при решении первой задачи. Трое из шести взрослых (пары А, В, Г) поступили совершенно одинаково: после непродолжительной ориентировки ребенка в числовой оси с указанием на 0,10 и 5 они предложили детям научиться считать от нуля (рисунок 1). Каждый из них ритмически переводил палец с риски на риску, называя 0, а 
потом побуждая ребенка к счету жестом и интонационным акцентом или вербальным вопросом («дальше?», «теперь?»). Вербальная составляющая при этом постепенно сокращалась, а жест оставался до конца. Еще одна мама (пара Е) попыталась научить ребенка именно этой простейшей стратегии, однако ребенок не дал ей этого сделать, предпочитая решать задачу своим путем: число 3 она определила как отличающееся на 2 вниз от числа 5 . Все описанные выше родители были мамами, папы вели себя немного подругому². Один папа (пара Д) сразу предложил ребенку считать за его указа-

Рисунок 1

Взрослый знакомит ребенка с расположением чисел на числовой прямой, предлагая пересчитать их вслед за дугообразными указательными жестами.

Видно, что ребенок задерживается на числе 5

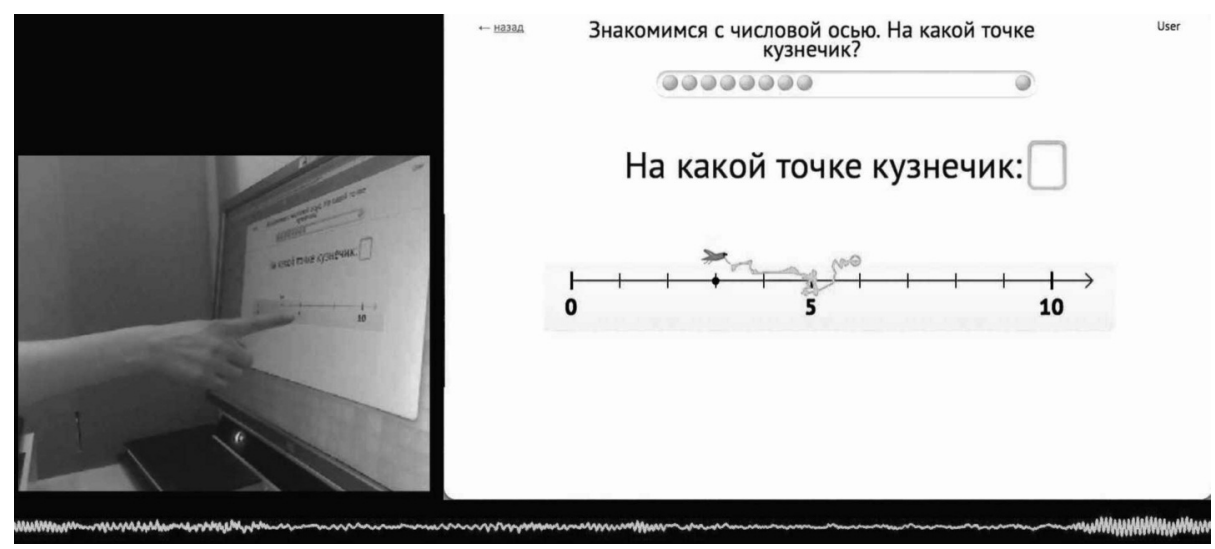

Рисунок 2

Взрослый указывает ребенку решение задачи, делая дугообразные движения пальцем

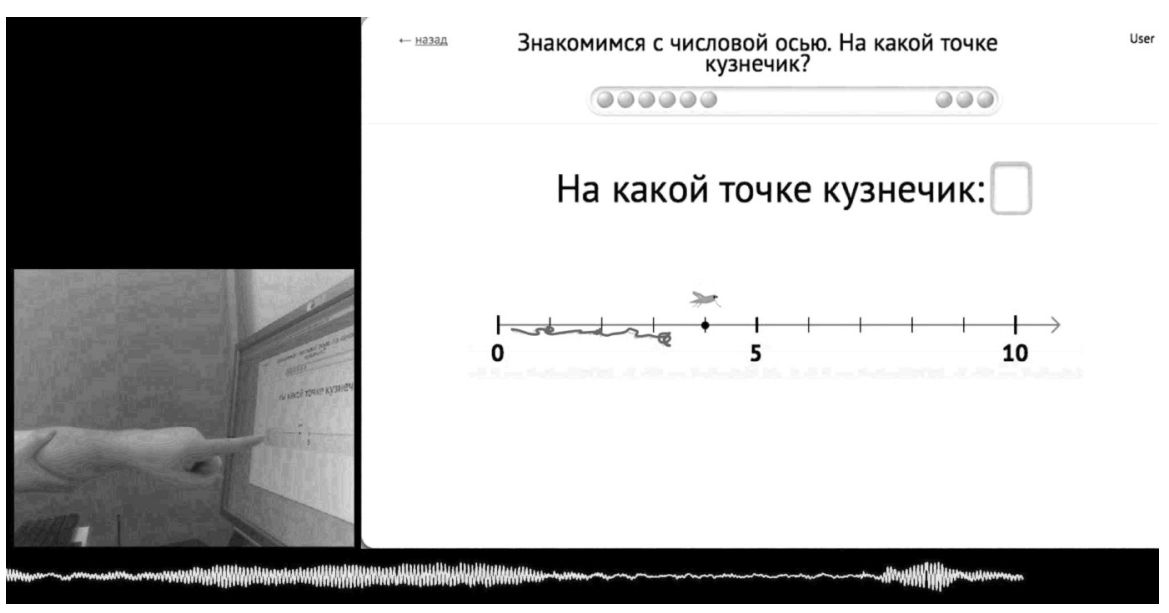

${ }^{2}$ Интересно, что поведение между мамами и папами сильно различалось, но для каких-либо обоснованных выводов, безусловно, нужна бо́льшая по объему выборка. 
тельными жестами, более того, методично предлагал это на протяжении всех восьми задач, что ребенок послушно и делал (рисунок 2). Другой папа (пара Б) вообще не давал никаких указаний в первой задаче и позволил ребенку разобраться самостоятельно. Таким образом, все родители, кроме одного, предложили своим детям именно стратегию 1: счет от числа 0 до целевой точки, несмотря на то что за все время эксперимента эта стратегия использовалась только одним взрослым один раз.

Лишь двое родителей попробовали предложить детям другие стратегии. Папа из пары Б, когда кузнечик сидел на точке 5, предложил использовать стратегию 9 - оценить точку 5 как середину между 10 и 0. Ребенок, проследив глазами вправо и влево по его указаниям, после этого посчитал от нуля до целевого числа (стратегия 1). Впоследствии при самостоятельном решении задачи он не стал использовать предложенную папой стратегию, а использовал снова стратегию 1. Мама из пары А после отсчета ребенка о числе 6 предложила считать от метки 5 (стратегия 5). Ребенок попробовал применить это в следующей задаче (число 9), однако мама предложила там считать от 10 вниз до числа (стратегия 4). Ребенок применил эту стратегию в этой и следующей задаче. Однако при самостоятельном решении этих задач предложенных родителем стратегий не использовал и считал до 8 и 9 от нуля (стратегия 1), хотя в некоторых других пробах использовал, помимо стратегии 1 , стратегии 2,5 и 7.

Обобщим результаты эксперимента по стратегиям обучения. В ходе совместной деятельности взрослые демонстрировали детям совсем не ту стратегию, которую использовали сами, но некоторый «базовый» способ действия, необходимый для овладения данной визуальной моделью: пересчет единичных интервалов (стратегию 1). Демонстрация же других способов, проделанная в редких случаях, не привела к усвоению детьми более сложных стратегий. Получается, что идеальная форма, существующая в культуре как способ решения перцептивной задачи взрослыми (Выготский, 2001), фактически не была развернута в интерпсихическом виде в ходе взаимодействия, как это предполагал Л.С. Выготский. В интерпсихическом взаимодействии мы увидели не способы действия взрослых, передаваемые ребенку в совместной деятельности, а сознательную реконструкцию взрослыми того способа действия, который необходим для овладения счетом и который может быть усвоен ребенком в ходе взаимодействия на этом этапе. Этот способ действия можно назвать специально выстроенной учебной формой. Однако это не значит, что другие способы действия оказались для ребенка недоступны: дети сумели на основе других знаний и представлений о числе дополнить базовый способ действия и сами применяли значительно более разнообразные стратегии, чем те, которые были предложены родителями. Ориентационные точки были выделены на стимульном материале и тем самым доступны непосредственному восприятию, которое могло повести за собой культурный способ действия с числовой осью. Кроме того, акцент на точках-ориентирах делали многие из родителей при первой презентации числовой прямой ребенку. 
Также дети считали не только вверх, как показывали им родители, но и вниз, и не только к целевой точке, но и от целевой точки.

Примечательно, что особенно сложной для освоения детьми оказалась стратегия 9, в которой оценивается относительное расстояние от ориентационных точек до целевого числа. Данная стратегия требует овладения принципиально иным свойством числовой оси, чем остальные стратегии. Если стратегии 1-8 опираются на представление о числовом ряде как упорядоченном наборе чисел, то для реализации стратегии 9 необходимо понимать числовую ось как отражающую пропорциональные отношения между числовыми интервалами, т.е. необходимо преодолеть сдвиг от представления о последовательности к представлению о пропорциональном соотношении (sequence-to-proportion shift), наблюдающийся, как правило, к третьему классу (Petitto, 1990).

Согласно данным нашего исследования, дети свободно восполняли и самостоятельно обогащали стратегии в рамках заданного родителями культурного способа действия - пересчета интервалов, однако принципиально иной способ оценки, основанный на соотношении интервалов, не был им доступен даже после указаний родителей. Учебная форма действия, выстраиваемая взрослыми, эффективна в том случае, если находится в зоне ближайшего развития ребенка. В противном случае, даже если ребенок следует за стратегией взрослого, он не включает ее в свои самостоятельные способы действия в дальнейшем.

\section{Выводы}

С помощью качественного микроанализа записей движений глаз, синхронизированных с аудио- и видеозаписями взаимодействия детей и их родителей, нами был детально рассмотрен процесс формирования культурного восприятия числовой прямой в качестве визуальной модели абстрактного числового ряда. Было проанализировано восприятие числовой прямой взрослыми, т.е. существующая в культуре идеальная форма восприятия, а затем прослежены пути передачи идеальной формы от взрослого к ребенку и после этого оценен результат обучения - сформированное у ребенка восприятие. Понятно, что подобный эксперимент лишь моделирует процесс освоения идеальной формы, который в экологичной ситуации разворачивается в течение длительного времени. Однако мы полагаем, что некоторые ключевые особенности могут быть выявлены уже в таком кратком прослеживании.

Во-первых, в ходе интерсубъективного взаимодействия идеальная форма не разворачивается в том виде, в каком она существует в культуре. Напротив, в ходе обучения воссоздается специфическая элементарная практика, учебная форма, которая считается доступной для ребенка и при этом позволяет надежно решить поставленную задачу, хотя и не оптимальным образом. По сравнению с этой базовой стратегией пересчета, которая спонтанно предлагалась ребенку в обучении, стратегии, используемые взрослыми, значительно гибче и вариативнее. Они наполнены эвристиками, проистекающими из более 
широких знаний о числовом ряде, чем знания, необходимые при простом пересчете.

Во-вторых, дети, в целом следуя за стратегиями, предложенными взрослыми, и интериоризируя их, как это предполагает Л.С. Выготский, одновременно существенно обогащают их и насыщают дополнительными способами действия, используя собственные предварительные знания о числовом ряде и его применении.

Положение о взаимодействии идеальной и реальной форм как источнике обучения и развития требует аккуратного пересмотра. Взаимодействие со взрослым действительно оказывает существенное влияние на возникающие у ребенка культурные формы восприятия, однако в ходе этого взаимодействия ребенок встречается не с идеальной, конечной формой восприятия взрослого, a c упрощенной формой поведения взрослого, специально созданной для ситуации обучения, учебной формой. Далее дети активно дополняют эту базовую стратегию, предложенную взрослыми. В итоге их самостоятельная форма восприятия приближается к идеальной форме восприятия взрослых по сравнению с той базовой учебной формой, которая была развернута в ходе взаимодействия со взрослым.

На примере анализа счета на числовой прямой можно сказать, что идеальная форма восприятия существует в скрытом виде: ребенку необходимо самостоятельно открывать ее, как используя предложенную взрослым базовую стратегию пересчета, так и встраивая конкретную задачу в интегральную систему знаний.

\section{Литература}

Выготский, Л. С. (2001). Лекции по педологии. Ижевск: Удмуртский университет.

Лурия, А. Р. (1962). Высшие корковые функции человека и их нарушения при локальных поражениях мозга. М.: Изд-во Московского университета.

Ссылки на зарубежные источники см. в разделе References после англоязычного блока.

Шварц Анна Юрьевна - постдокторант, Институт математического и научного образования им. Г. Фройденталя, Утрехтский университет (Нидерланды); старший научный сотрудник, факультет психологии, МГУ имени М.В. Ломоносова, кандидат психологических наук.

Сфера научных интересов: культурно-исторический подход, энактивизм, воплощенное познание, теоретическое восприятие, визуализация, визуальные модели, математическое мышление, психология математического образования, движения глаз.

E-mail: shvarts.anna@gmail.com 


\title{
Does the Real Form Interact with the Ideal Form? A Study of the Teaching-Learning to Count on the Number Line by Means of Eye-Tracking
}

\author{
A.Yu. Shvarts ${ }^{\mathrm{a}, \mathrm{b}}$ \\ ${ }^{a}$ Utrecht University, 5 Princetonlaan, 3584 CC, Utrecht, Netherlands \\ ${ }^{b}$ Lomonosov Moscow State University, 1 Leninskie Gory, Moscow, 119991, Russian Federation
}

\begin{abstract}
The article investigates acquisition of mathematical knowledge in collaboration with an adult as it is exemplified by preschoolers' learning to count on the number line. A qualitative analysis of the eye-movements reveals the diversity of possible strategies in determination of a number on the number line. The developmental experiment discloses the mechanisms of emergence of these strategies in children. The quantitative comparison of the adults' strategies, the strategies, which are involved in the teaching-learning process, and the strategies that the children used after the learning stage demonstrates the process of development $\left(\chi^{2}=44.936\right.$; $p<.001)$. We distinguished the statistically significant differences between the stages in the ratio of counting up versus down along the number line and in the ratio of counting from versus towards the target point. The results demonstrate that children's strategies after the learning stage are more similar to the adults' inherent strategies than to the strategies that were introduced by the adults during the teaching stage. The analysis of the videos of shared activity that was synchronized with the eye movements showed that the adults demonstrated the basic strategy to the children at the teaching phase as they guided children's perception by their pointing gestures and speech. However, the adults did not expose the ideal form, namely the diversity of their own strategies during their teaching. Nevertheless, the children were able to supplement the given teaching/learning form of counting from zero up along the number line to the target point with a variety of strategies by themselves, relying on their coherent notion of the number concept. The strategy that required the sequence-to-proportion shift was the only one that children were not able to constitute by themselves. According to our results, the ideal, cultural form of perception exists in the latent form, and a child needs to re-constitute it in their own practice. The children rely on the basic strategy and enrich this strategy as they include it in the integral conceptual knowledge about numbers. The results enrich our understanding of microgenesis of mathematical knowledge during the collaboration with an adult and open perspective on learning as an active reinvention of ideal form on the ground of cultural practice.
\end{abstract}

Keywords: cultural-historical approach, Vygotsky, mathematics education, teaching, learning, ideal form, shared activity, number line, mathematical concept, eye-tracking, oculography.

\section{References}

Bjuland, R. (2012). The mediating role of a teacher's use of semiotic resources in pupils' early algebraic reasoning. ZDM, 44(5), 665-675. http://doi.org/10.1007/s11858-012-0421-2 
Dehaene, S., Bossini, S., \& Giraux, P. (1993). The mental representation of parity and number magnitude. Journal of Experimental Psychology: General, 122(3), 371-396. doi:10.1037/00963445.122.3.371

Duval, R. (2006). A cognitive analysis of problems of comprehension in a learning of mathematics. Educational Studies in Mathematics, 61(1-2), 103-131. doi:10.1007/s10649-006-0400-z

Fias, W. (1996). The importance of magnitude information in numerical processing: Evidence from the SNARC Effect. Mathematical Cognition, 2(1), 95-110. doi:10.1080/135467996387552

Gelman, R., \& Gallistel, C. R. (1978). The child's understanding of number. Cambridge, MA: Harvard University Press.

Goldin-Meadow, S. (1999). The role of gesture in communication and thinking. Trends in Cognitive Sciences, 3(11), 419-429.

Heine, A., Thaler, V., Tamm, S., Hawelka, S., Schneider, M., Torbeyns, J., ... Jacobs, A. M. (2010). What the eyes already "know": Using eye movement measurement to tap into children's implicit numerical magnitude representations. Infant and Child Development, 19(2), 175-186. doi:10.1002/icd.640

Hitt, F. (1998). The role of the semiotic representations in the learning of mathematics. Proceedings of the British Society for Research into Learning Mathematics, 18(3), 23-28. Retrieved from http://www.bsrlm.org.uk/wp-content/uploads/2016/02/BSRLM-IP-18-3-4.pdf

Hubbard, E. M., Piazza, M., Pinel, P., \& Dehaene, S. (2005). Interactions between number and space in parietal cortex. Nature Reviews Neuroscience, 6(6), 435-448. doi:10.1038/nrn1684

Link, T., Huber, S., Nuerk, H.-C., \& Moeller, K. (2014). Unbounding the mental number line - new evidence on children's spatial representation of numbers. Frontiers in Psychology, 4, 1021. doi:10.3389/fpsyg.2013.01021

Loetscher, T., Bockisch, C. J., \& Brugger, P. (2008). Looking for the answer: The mind's eye in number space. Neuroscience, 151(3), 725-729. doi:10.1016/j.neuroscience.2007.07.068

Luria, A. R. (1962). Vysshie korkovye funktsii cheloveka $i$ ih narusheniya pri lokal'nyh porazheniyah mozga [Higher cortical functions in man and thier disturbances in the presence of local brain lesions]. Moscow: Moscow University Press. (in Russian)

Petitto, A. L. (1990). Development of numberline and measurement concepts. Cognition and Instruction, 7(1), 55-78.

Presmeg, N., Radford, L., Roth, W.-M., \& Kadunz, G. (2016). Semiotics in mathematics education. Dordrecht, The Netherlands: Springer. doi:10.1007/978-3-319-31370-2

Radford, L. (2010). The eye as a theoretician: seeing structures in generalizing activities. For the Learning of Mathematics, 30(2), 2-7.

Roth, W.-M. (2008). The dawning of signs in graph interpretation. In L. Radford, G. Schubring, \& F. Seeger (Eds.), Semiotics in mathematics education: Epistemology, history, classroom, and culture (pp. 83-102). Rotterdam: Sense.

Schneider, M., Heine, A., Thaler, V., Torbeyns, J., De Smedt, B., Verschaffel, L., ... Stern, E. (2008). A validation of eye movements as a measure of elementary school children's developing number sense. Cognitive Development, 23(3), 409-422. doi:10.1016/j.cogdev.2008.07.002

Siegler, R. S., \& Opfer, J. E. (2003). The development of numerical estimation. Psychological Science, 14(3), 237-250. doi:10.1111/1467-9280.02438

Sullivan, J. L., Juhasz, B. J., Slattery, T. J., \& Barth, H. C. (2011). Adults' number-line estimation strategies: evidence from eye movements. Psychonomic Bulletin and Revier, 18(3), 557-563. doi:10.3758/s13423-011-0081-1 
Vygotsky, L. S. (2001). Lektsii po pedologii [Lectures on pedology]. Izhevsk: Udmurt State University.

Anna Y. Shvarts - postdoctoral researcher, Freudenthal Institute for Science and Mathematics Education, Faculty of Science, Utrecht University; senior research fellow, Lomonosov Moscow State University, Ph.D.

Research area: culture-historical approach, enactivism, embodied cognition, theoretical perception, visualization, visual models, mathematical thinking, psychology of mathematics education, eye movements.

E-mail: shvarts.anna@gmail.com 